\title{
Diversité et recomposition de l'espace rural dans le district d'Amatola, province du Cap de l'Est
}

\author{
Laurent, Lhopitallier \\ Institut français d'Afrique du Sud, Po Box 542, Newtown 2113, République Sud-Africaine \\ Patrick Caron \\ Centre de coopération internationale en recherche agronomique pour le développement, \\ Département territoire, environnement et acteurs, CIRAD/TERA, BP 5035, 34032 Montpellier
}

\begin{abstract}
RÉSUMÉ. - Un modèle d'organisation de l'espace et des dynamiques spatiales d'un district d'Afrique du Sud montre les espaces bien délimités et dissymétriques issus de la politique d'apartheid. La distribution spatiale de la population et des centres urbains et industriels et les mouvements migratoires répondent à un phénomène de gravité centré sur East London. Il existe une hiérarchie de centres secondaires et tertiaires, gravitant autour d'East London. Les voies de communication et la hiérarchie des bourgs et petites villes dessinent un treillage qui correspond rarement aux limites administratives. Les flux d'hommes et de femmes, de marchandises, de capitaux et d'informations révèlent le fonctionnement d'interdépendances et l'existence de réseaux complexes et enchevêtrés. L'analyse permet d'identifier des mesures d'aménagement du territoire nécessaires pour corriger l'héritage de la politique de développement séparé.
\end{abstract}

AFRIQUe du Sud, AMATOLA, AMÉNAGEMENT DU TERRTTORE, BANTOUSTAN, ZONAGE

Le district d'Amatola est situé dans la province de l'Eastern Cape, dans une région où les anciens bantoustans du Ciskei et du Transkei jouxtaient la République sud-africaine.
ABSTRACT. - Eiversity and recomposition of rural space in the district of Amatola, Eastern Cape Province.- A model of spatial organisation and dynamics in a South African district shows up clearly delimited, dissymetrical spaces produced by the policy of apartheid. Migratory movements and the spatial distribution of the population and of the urban and industrial centres highlight a gravity pattern, with a hierarchy of secondary and tertiary centres gravitating around East London. The limites of administrative entities are a poor fit to the lattice formed by the communication routes and the hierarchy of towns. Flows of people, goods, capital and information reveal an interdependence and the existence of complex, entangled networks. The analysis points up the regional development measures needed to correct the legacy of a segregated development policy.

Amatola, Bantustan, Regional deVelopment, SOUth AFrica, ZONING

Depuis l'abolition du régime d'apartheid, ce district est animé de profondes recompositions politiques, sociales, spatiales et économiques. En 1995, l'État a procédé à un 
redécoupage administratif et précisé les enjeux d'aménagement du territoire et de redistribution des richesses (Ministry for Provincial Affairs and Constitutionnal Development, 1998). Pour corriger le lourd héritage de la politique de déve loppement séparé, la délimitation du district d'Amatola (environ $21000 \mathrm{~km}^{2}$ ) comme en de nombreuses régions d'Afrique du Sud, a transcendé les «frontières» de l'apartheid.

Pour répondre à ces défis, le district dispose d'une administration et de services techniques. Le Conseil de district a pour mandat d'assurer le développement des zones rurales Il offre des services aux autorités locales ${ }^{1}$ qui n'ont pas, dans l'Eastern Cape, de budget propre. La direction du Conseil est composée de membres élus par les différentes autorités locales. Ses ressources proviennent des subventions des gouvernements national et provincial et du prélèvement de taxes locales. Son rôle est amené à s'effacer au fur et à mesure que les autorités locales gagneront en capacité financière et organisationnelle ${ }^{2}$.

Un zonage « à dires d'acteurs » a été réalisé ${ }^{3}$ en s'appuyant sur une méthodologie conçue et expérimentée au Brésil (Caron, 1997). L'organisation d'un ensemble d'informations et de connaissances, formalisées ou non, cherche à rendre compte de la diversité des situations actuelles et des dynamiques qui les animent. L'application de cette proposition dans le contexte sud-africain avait pour objectif, d'une part, d'identifier les principes d'organisation de l'espace dans une perspective d'aménagement du territoire, d'autre part, de préciser le contexte et les conditions de mise en cuvre d'opérations de développement local (Bonnal et al., 1998).

1. Ce sont les Transitionnal Representative Councils : représentations du Conseil de district dans les Magisterial Districts, composée de membres élus par la communauté et d'un administrateur nommé par le Conseil de district. Sans pouvoir exécutif, ils ont un rôle consultatif auprès du Conseil.

2. La situation actuelle des collectivités locales et territoriales est transitoire. De nouvelles structures seront mises en place après les élections locales de 1999.

3. L'équipe était composée de Patrick Madikizela (Agricultural Research Council), Nobuntu Mapeyi (Agricultural and Rural Development and Research Institute), Kuthala Tolbat (Eastern Cape Department of Agriculture and Land Affairs), Florence Lasbennes (Centre national d'études agronomiques en régions chaudes / CIRAD), Mehdi Saqqali (IEDES-CIRAD), Laurent Lhopitallier (IFAS) et Patrick Caron (CIRAD).

\section{La méthodologie}

\section{Les options refenues}

Les données disponibles dans la littérature sont souvent sectorielles (pédologie, climatologie, démographie, volumes de production, santé, etc.) et rarement d'actualité. Elles rendent mal compte des dynamiques du monde rural. Quand ils existent, les recensements ${ }^{4}$ s'intéressent à la description de variables par rapport à des limites administratives qui, si elles ont un sens pour l'action publique, ne sont pas forcément pertinentes pour comprendre les évolutions et les perspectives de la production agricole. Deux options ont donc été retenues. La première consiste à valoriser les savoirs de "personnes ressources » ayant une bonne connaissance du milieu pour y vivre et y travailler. Le choix des variables qui expliquent et rendent compte de la diversité et de la dynamique des situations n'est pas déterminé a priori mais devient l'objet des enquêtes (Perrot et Landais, 1993). Il n'est pas lié au domaine de compétence de l'expert chargé de l'analyse. C'est à partir des dires de ces acteurs, qui ont déjà consciemment ou non réalisé l'essentiel du travail d'analyse des situations rurales complexes, qu'est structurée la production de connaissances. La seconde consiste à retenir le support cartographique comme base de dialogue et de représentation des connaissances: il permet aux personnes enquêtées de s'exprimer en faisant référence à des lieux précis, à des objets matériels, à des limites physiques, etc. Grâce à ce support, on caractérise la diversité et la dynamique spatiales, formulées sous forme d'une nouvelle représentation cartographique.

Le principe est de représenter sur une carte synthétique la diversité de l'espace étudié, compris comme un produit social (Brunet, 1987). Comprendre ensuite la diversité des milieux, répondre à la question « pourquoi ceci se trouve-til là et pas ailleurs, là plus qu'ailleurs ?» conduit à l'analyṣe de l'organisation et de I'évolution de cet espace. Pour modéliser ${ }^{5}$ la complexité des situations, plusieurs types

4. Les recensements étaient très rares et peu fiables dans les exbantoustans. Les résultats du premier recensement de la « Nouvelle Afrique du Sud» conduit en 1996 ne sont encore que partiellement disponibles.

5. La modélisation ne constitue pas ici une démarche normative. Il s'agit de la construction de modèles, compris comme des « repré- 


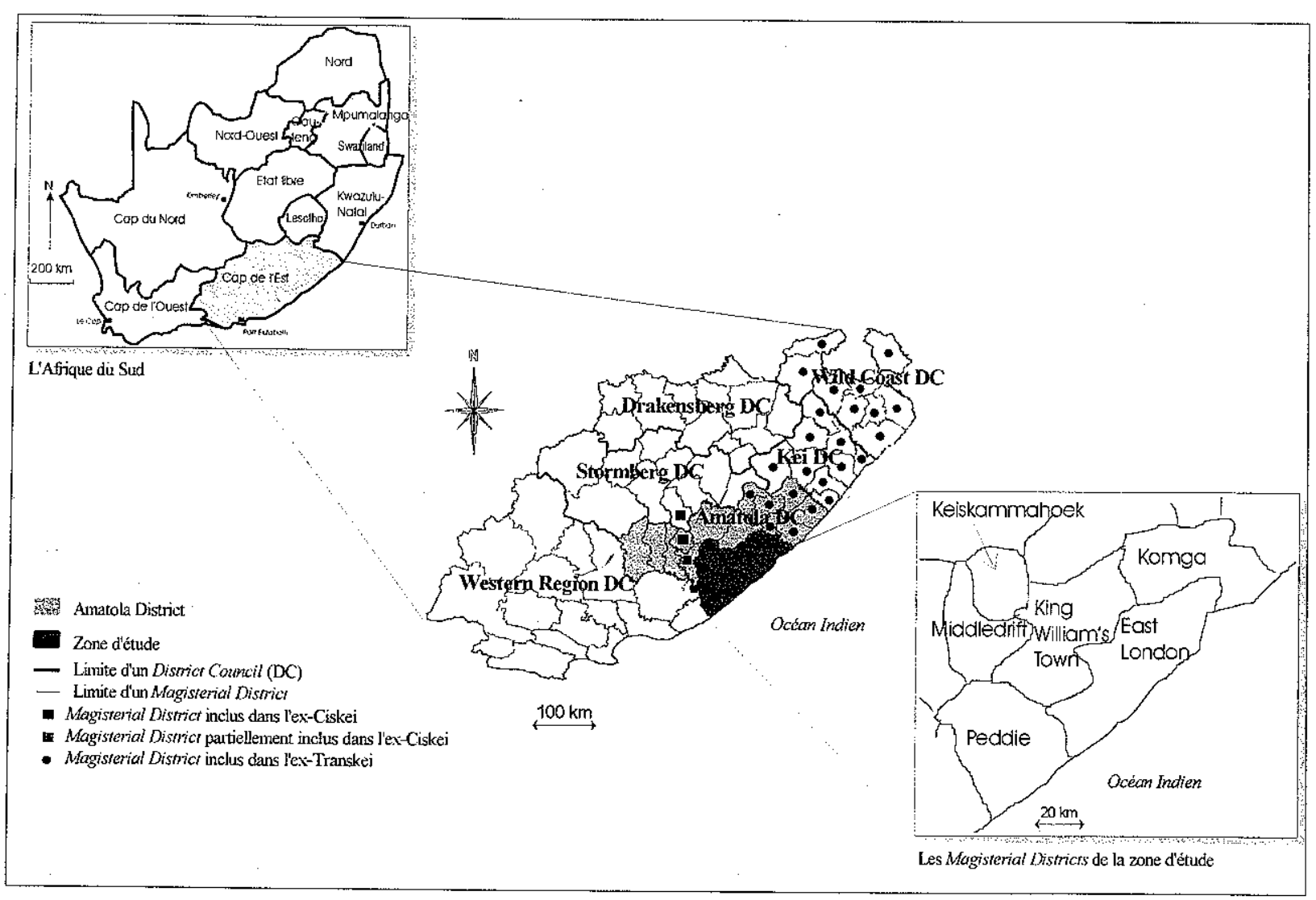

Fig. 1.-Carte de la province de l'Eastern Cape : zone d'étude et limites des Magisterial Districts et des Districts Councils.

d'informations sont mobilisés, et tout d'abord les représentations que les acteurs se font de la réalité. Elles permettent de caractériser la diversité des espaces et les facteurs qui l'expliquent ou la révèlent. Elles sont systématisées au cours d'enquêtes avec des personnes ayant une connaissance de tout ou partie de la zone d'étude. L'étude est ensuite réalisée grâce à l'analyse comparative de leurs dires, complétée par l'observation directe des paysages et des activités humaines et les données secondaires statistiques, bibliographiques ou cartographiques concernant les ressources naturelles, les infrastructures, la démographie, etc.

La méthodologie s'appuie sur la notion d'unité spatiale homogène (USH), définie comme une unité spatiale au

sentations intelligibles artificielles, symboliques, des situations dans lesquelles nous intervenons [... et...] que l'on construit dans sa tête » (Le Moigne, 1990). sein de laquelle les ressources productives, leur utilisation, leur mise en valeur par les acteurs et les difficultés rencontrées constituent une problématique homogène, dont la variabilité est minime à l'échelle retenue. Six étapes méthodologiques sont définies (Caron, 1997) : études bibliographique et cartographique, sélection du document de travail, élaboration du guide d'entretien et choix des informateurs; entretiens; analyse comparative des entretiens et identification d'USH; intégration des données des entretiens et des informations secondaires; analyse et caractérisation de l'organisation et du fonctionnement de l'espace étudié et des tendances d'évolution spatiale et sociale; restitution aux acteurs.

Le rôle du chercheur chargé de l'élaboration de la carte n'est pas neutre. Il existe plusieurs possibilités de représenter un même espace à partir des mêmes dires d'acteurs. Une définition préalable et précise des objectifs attendus est 
indispensable. Par ailleurs, la prise en compte des dynamiques territoriales macro-régionales ou nationales n'est pas aisée: ni ces dynamiques, ni les enjeux ne peuvent être tous explicités.

\section{La mise en œuvre de la méthodologie}

La zone d'étude (fig. 1) correspond à une partie du district d'Amatola. Cette étude visait en effet à tester la démarche, avant d'envisager son extension, avec les services du Conseil du district, à l'ensemble du district. Pour constituer un ensemble spatial diversifié, la zone d'étude a été définie comme un ensemble de Magisterial Districts ${ }^{6}$, comprenant les principaux centres urbains, une frange du littoral océanique, une partie de l'ancien Ciskei et une partie de l'ancien «corridor», le couloir séparant le Ciskei du Transkei occupé principalement par de grandes fermes commerciales «blanches». Cette zone recouvre une superficie totale d'environ $7500 \mathrm{~km}^{2}$. Elle est totalement incluse dans le district d'Amatola, défini lors du dernier découpage administratif, après l'abolition de l'apartheid, par agrégation de 19 Magisterial Districts.

Les personnes interrogées ont été contactées via les Transitional Representative Councils (TRC). Nombre d'entre elles étaient des membres du TRC, mais le choix s'est également porté sur d'autres interlocuteurs ${ }^{7}$, représentants d'organisations et de catégories socioprofessionnelles diversifiées (profession agricole, instituteurs, représentants des ordres religieux, commerçants, etc.).

6. Le Magisterial District est une unité administrative héritée de la colonisation anglaise, d'une superficie comprise entre 500 et $1500 \mathrm{~km}^{2}$. Il n'est cependant pas doté de pouvoirs exécutifs et ses représentants ne sont pas élus, mais nommés par le gouvernement. Sorte de mini-préfecture, il assure le lien entre les populations dans sa juridiction et le gouvernement central, essentiellement dans le domaine judiciaire. Les Magisterial Districts d'East London (regroupant également l'ancien Magisterial District de Mdantsane), de King William's Town (regroupant également l'ancien Magisterial District de Zwelitsha), de Komga, de Keiskammahoek, de Middledrift et de Peddie ont été choisis.

7. Ces personnes sont essentiellement choisies pour leur connaissance approfondie du district acquise à travers leurs activités professionnelles ou extraprofessionnelles.
Le support de travail est un assemblage de quatre cartes topo-cadastrales du Chief Directorate of Survey, à l'échelle de $1 / 250000$, datant de 1987 . Y figurent la localisation des villages et des villes, les routes et les pistes, les cours d'eau, les courbes de niveau, les forêts et réserves naturelles et les limites administratives.

Un guide d'enquête ouverte a été élaboré. Deux critères initiaux d'identification de la diversité ont été retenus, pour permettre d'engager le dialogue. Outre les activités productives, il a été décidé de prêter une attention particulière aux sources de revenus, compte tenu des caractéristiques démographiques des zones rurales des anciens bantoustans (population essentiellement composée de très jeunes et de personnes âgées) et de l'importance des revenus de la migration et des pensions et retraites. Le guide d'enquête comprend différentes rubriques (réalités observées, facteurs explicatifs, perspectives).

Chaque enquête ${ }^{8}$ est conduite avec des groupes de 3-4 personnes. Elle porte sur une zone correspondant approximativement au Magisterial District afin de permettre une analyse comparative des dires d'acteurs. La zone d'enquête n'est cependant pas bornée aux limites administratives, ce qui permet ultérieurement de vérifier les cohérences et les continuités d'une zone d'enquête à l'autre.

Par la mise en évidence des cohérences (et incohérences) entre les cartes obtenues au cours de chacune des enquêtes, l'analyse comparative des résultats permet dans un premier temps de dessiner une carte synthétique pour chaque Magisterial District. Une nouvelle carte de la zone d'étude est ensuite produite, en vérifiant cohérences et incohérences sur les zones de recouvrement, en agrégeant des USH parfois distantes mais présentant des caractéristiques similaires et en homogénéisant les appellations des USH. L'ensemble des informations recueillies est organisé pour caractériser chaque unité.

8. Les personnes interrogées travaillent sur une carte recouverte de papier calque. Dès qu'elles parviennent à se repérer sur le support cartographique, l'enquêteur les incite à tracer des limites et à définir les caractéristiques de zones qui leur semblent homogènes selon les deux critères retenus. À la fin de l'enquête et sur la base des caractéristiques de chaque zone homogène, enquêteur et enquêtés reviennent de manière critique sur le découpage initial, quitte à le modifier. 


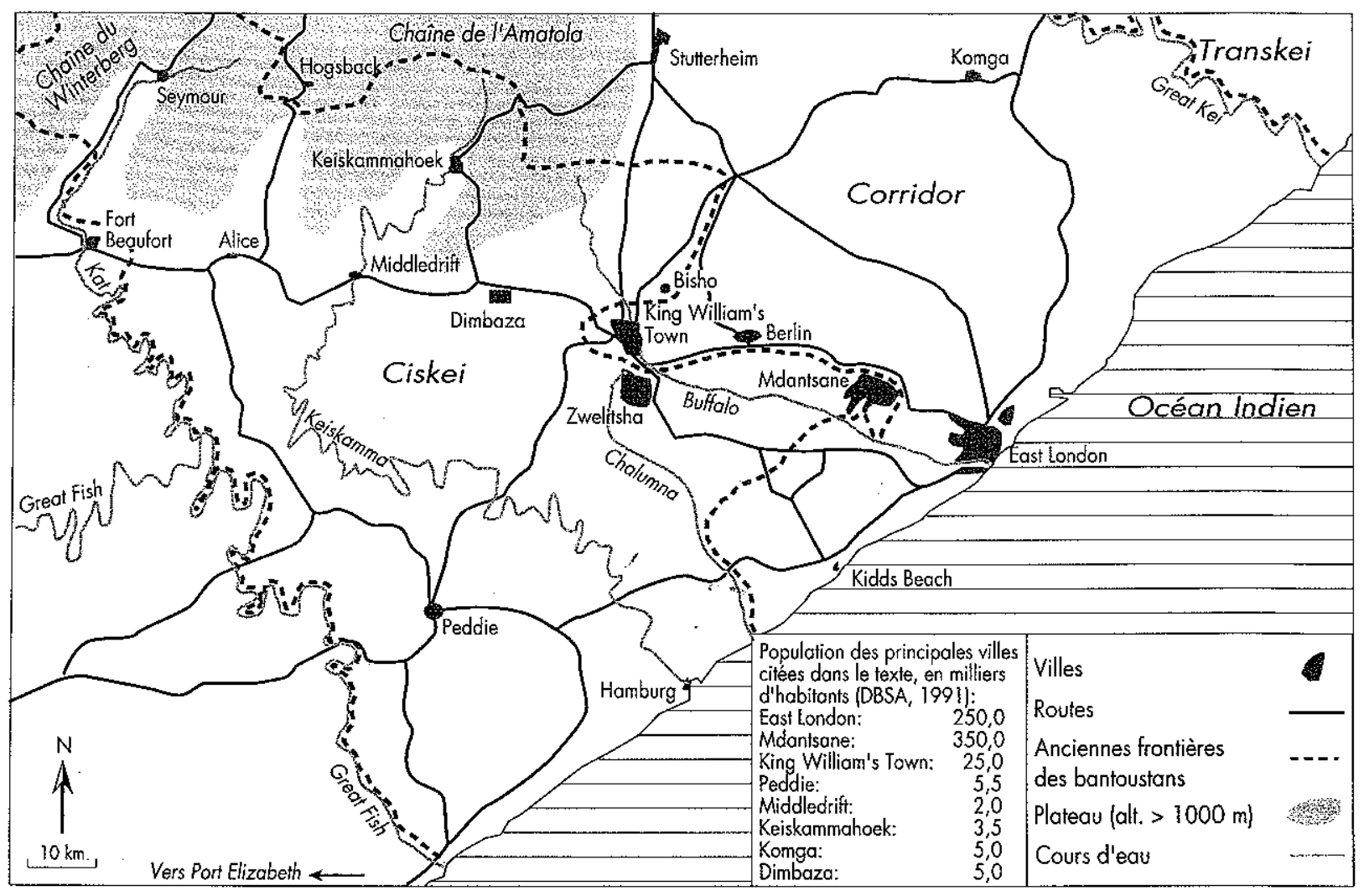

Fig. 2.-Zone d'étude.

\section{La diversité des espaces ruraux}

Au début des enquêtes, les personnes interrogées tiennent généralement un discours du type : "Tout est pareil, l'essentiel des revenus provient des allocations de vieillesse.» Par la suite, la carte se révèle être un excellent support de communication pour mettre en évidence la diversité des situations, même si celle-ci fait appel à des éléments qui n'y figurent pas (les conditions de transport pour aller travailler tous les jours en ville par exemple). Neuf zones ont été identifiées, constituées chacune de l'agrégation de plusieurs USH (fig. 3).

1. La zone urbaine et sa périphérie. - Elle comprend les principaux centres urbains ${ }^{9}$ (East London), les villes secondaires (conurbation King William's Town-ZwelitshaBisho), les villes tertiaires ${ }^{10}$ et les bourgs ruraux, les townships créés pour accueillir les populations noires déplacées, les zones industrielles et les zones «rurbaines». Ces dernières sont constituées par les villages d'où les ouvriers peuvent aller quotidiennement travailler en ville ou dans les

9. Il existe une hiérarchie implicite entre les différents centres urbains, qui repose sur le type de services offerts et sur l'intensité des phénomènes d'attraction. Ainsi, East London est la grande métropole de la région, les villes dites secondaires offrent un éventail assez large de services, les villes tertiaires offrent, elles, un service minimum (la poste, une banque, une supérette). Les bourgs ruraux sont de simples gares routières urbanisées avec quelques boutiques. Ils sont l'héritage des anciens centres administratifs coloniaux britanniques.

10. Ces villes connaissent une multiplication des camps de squatters dans les espaces interstitiels urbains (friches industrielles, ancien commonage). Ces camps sont occupés par des populations des campagnes avoisinantes qui se rapprochent des centres urbains pour plusieurs raisons : facilité d'accès aux services (éducation, électricité, adduction d'eau); offres d'emploi; grandes voies de communication. 


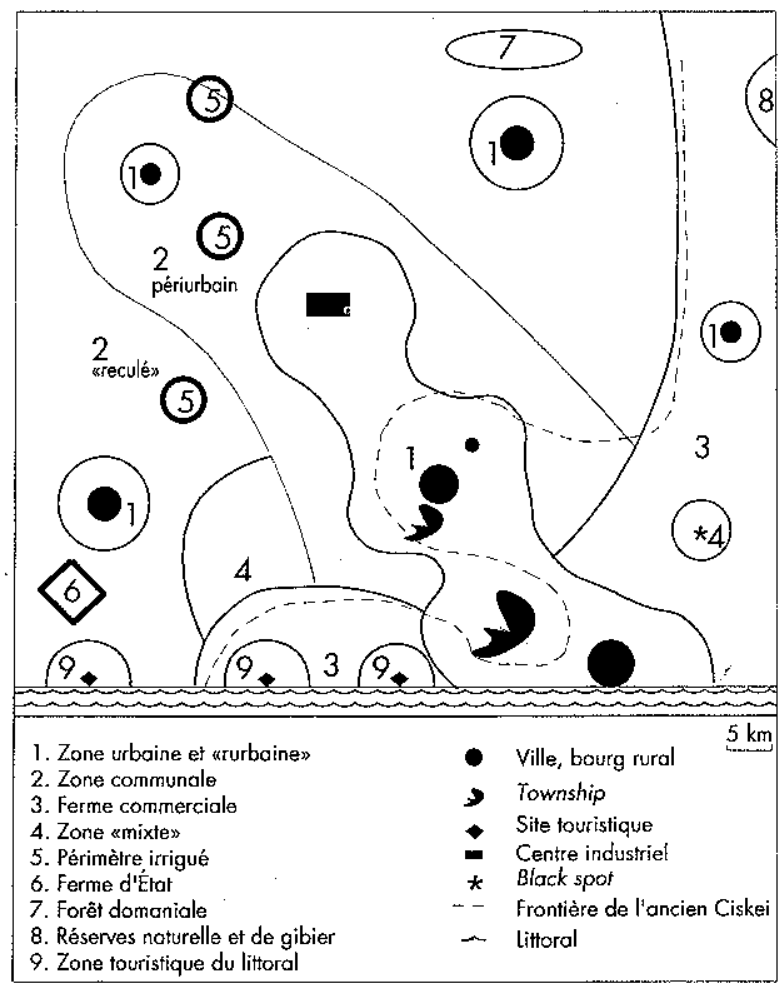

Fig. 3.-Diversité et modèle d'organisation de l'espace rural dans la zone d'étude.

zones industrielles, grâce à l'organisation des transports collectifs privés (taxis) et publics (bus, train). Les revenus de ces emplois constituent le principal apport monétaire pour la majorité de la population, qui s'apparente de ce fait à un prolétariat urbain (lieu de travail, style de vie) résidant en milieu rural. Cette zone est faite de deux ensembles : (I) un continuum territorial qui s'étend depuis East London en direction du nord-ouest le long de la rivière Buffalo, caractérisé par l'existence d'axes de communication majeurs (routes, chemin de fer) et la succession de centres urbains et industriels; (II) des alvéoles centrées sur les bourgs ruraux, reliées entre elles et au continuum précédent par un réseau routier goudronné.

La crise du logement dans les villes des anciens bantoustans est aussi l'une des raisons du développement de ces camps de squatters, où logent notamment de nombreux fonctionnaires, n'ayant pas trouvé ou ne pouvant plus s'offrir de logement en ville.
2. Les zones communales.-- Elles sont constituées de villages de l'ancien Ciskei où l'usage collectif des ressources foncières s'est maintenu. On y rencontre des zones de montagne au nord, sur le premier contrefort du High Veld, caractérisées par l'importance du paysannat, des zones périurbaines où emploi urbain et production agricole pour l'autoconsommation se complètent, des zones «reculées» (remote) où les transferts sociaux et les migrations à longue distance vers les grands centres urbains et les mines (Johannesburg, Le Cap) offrent les principales sources de revenus.

3. Les fermes commerciales.- La plupart des fermes commerciales appartiennent à des producteurs blancs. Elles sont situées dans les zones de pluviométrie élevée (ancien corridor et proximité du littoral vers l'ouest). Ces zones sont généralement traversées par des axes de communication majeurs. De nombreuses fermes emploient des ouvriers agricoles noirs. Toutefois, certains producteurs noirs se sont installés dans les années 1970-1980 dans le Magisterial District de King William's Town, après avoir été expropriés du corridor (déplacement des black spots). En fonction des choix et techniques de production agricole, on peut distinguer une aire de production intensive de lait et de fruits, souvent irriguée, le long des axes routiers et près des centres urbains, une aire de production extensive ou ultra-extensive (grandes exploitations et propriétaires absentéistes) de bovins de boucherie, des exploitations irriguées d'agrumes ou de production maraîchère à proximité des rivières.

4. Des zones mixtes : fermes commerciales et villages communaux.- On observe deux cas, très différents l'un de l'autre. Le premier, dans l'ancien corridor, est une zone de production extensive semblable à la zone 3 , mais au sein de laquelle il existe encore des villages communaux (black spots). Leurs habitants travaillent généralement dans les fermes voisines. Depuis 1994, tensions et conflits sont vifs et certaines fermes sont mises en vente. Le second cas correspond à une zone d'anciennes fermes commerciales de production d'ananas, rachetées lors de la création de l'État du Ciskei (1981) et louées à des fonctionnaires absentéistes. Elles sont plus ou moins exploitées à des fins pastorales. La charge animale est faible et l'on note un embroussaillement important des parcelles. Tensions et conflits sont nombreux entre les populations des villages situés dans la zone et les propriétaires absentéistes. 
5. Les périmètres irrigués de l'ancien Ciskei.-- Il s'agit d'un chapelet d'unités spatiales situées le long des rivières Fish ou Keiskamma, ou de l'un de leurs affluents. Les périmètres ont été mis en place par l'État du Ciskei et gérés par une entreprise para-étatique. Choix de production, itinéraires techniques, approvisionnement en intrants et commercialisation des produits ont été imposés aux paysans. Depuis 1994, des tentatives de transfert à des opérateurs privés ont vu le jour, en particulier aux paysans qui les exploitaient auparavant. Cette transition ne s'opère pas sans problèmes et l'on note sur certains périmètres une déprise sensible et une dégradation des équipements et du matériel agricoles. Selon les choix de production antérieurs, on distingue des périmètres de production d'agrumes, de lait ou de légumes.

6. Les fermes de production d'ananas de l'ancien Ciskeí.Ces fermes ont été mises en place par l'État du Ciskei et gérées par une entreprise d'État, après l'expropriation de producteurs blancs dans le Magisterial District de Peddie. Elles s'étalent sur plusieurs milliers d'hectares. Depuis 1994, après des tentatives infructueuses de transfert à des opérateurs privés, cette zone est le siège de conflits majeurs. Elle est envahie par les ouvriers agricoles qui n'ont plus été payés depuis 1994 et par les paysans des villages voisins qui en revendiquent la propriété. Les bovins pâturent les plantations d'ananas abandonnées. Les équipements et le matériel agricoles sont endommagés.

\section{Les forêts domaniales et plantations forestières.- Pour} la plupart localisées dans les Magisterial Districts de Keiskammahoek et de King William's Town, elles appartenaient à l'État du Ciskei. Dans le cas des forêts domaniales, les habitants des villages voisins peuvent en extraire le bois mort et y envoyer pâturer leurs animaux. Dans le cas des plantations forestières, les emplois de gardes forestiers se font de plus en plus rares depuis 1994.

8. Les réserves naturelles et de gibier.-Il existe une réserve naturelle publique dans le district d'East London. Par ailleurs, certaines fermes commerciales se reconvertissent vers l'élevage de gibier dans le Magisterial District de Komga ${ }^{11}$. Dans le Magisterial District de Victoria East (hors

11. Le problème du vol de bétail s'étant considérablement aggravé depuis 1994; les exploitants organisent des rondes de de la zone d'étude), a également été mise en place une réserve de gibier sauvage, comprenant la partie nord du Magisterial District de Peddie. Le développement des activités touristiques est limité par l'enclavement, l'accès difficile à cette zone et la réputation d'insécurité dont les anciens bantoustans souffrent toujours.

9. La zone littorale.-Villes, villages et lieux touristiques se sont développés le long du littoral, y compris dans l'ancien Ciskei, où le gouvernement du bantoustan avait attribué une concession au groupe d'entrepreneurs de jeux et de casinos Sun. Ces zones offrent des emplois pour les populations des villages voisins situés le long de l'axe routier East London-Port Elizabeth dans le Magisterial District de Peddie. Les revenus des emplois dans le secteur du tourisme et des services sont importants dans ces villages qui deviennent parföis de véritables bourgs ruraux.

\section{Un modèle d'organisation de l'espace régional}

Pour élaborer un modèle graphique d'organisation de l'espace, nous avons choisi de représenter dans un premier temps ses structures élémentaires, au nombre de quatre (fig. 4 et paragraphes ci-dessous). Leur superposition fournit une représentation globale de l'organisation de l'espace rural et de sa diversité (fig. 3).

\section{Les structures élémentaires de l'espace}

1. La marque de la politique d'apartheid.--L'abandon de la politique d'apartheid, en partie relayée par le gouvernement du Ciskei, est récent. L'empreinte de l'apartheid dans les paysages et l'organisation de l'espace rural se ressent encore fortement. Elle se traduit par l'appropriation des ressources naturelles les plus favorables à l'agriculture (pluviométrie

surveillance collective autour de leurs propriétés, jour et nuit. $\mathrm{Ce}$ phénomène, associé à la baisse des revenus de l'élevage, conduit un nombre grandissant d'exploitants à se reconvertir vers l'élevage de gibier (antilopes essentiellement), destiné à la production de viande, mais aussi à l'organisation de chasses pour des étran gers. Afin de diminuer les coûts liés à la mise en place de clôtures adaptées, les propriétaire ont tendance à se regrouper en Conservancies et à mettre ainsi en commun leurs ressources foncières. 
élevée, topographie peu accidentée pour la mécanisation, etc.) et l'installation de fermes commerciales blanches dans le corridor et la zone littorale en direction de l'ouest (ancien bassin de production de l'ananas) (fig. 4). Bien sûr, les caractéristiques des ressources naturelles ne sauraient expliquer à elles seules la distribution ethnique des espaces. Même si elles leur sont en partie liées, les guerres passées et la localisation par rapport aux centres urbains et aux axes de communication ont également joué un rôle important. La différenciation des régimes fonciers est radicale : propriété individuelle dans les zones commerciales; régimes mixtes et extrêmement complexes dans les zones communales, imposés par les administrations successives comme autant de strates donnant aujourd'hui lieu à de nombreux conflits et revendications. Dans le second cas, on distingue des terres tribales, des terres en trust (propriété de l'État) ou en propriété privée. En fait, la situation est beaucoup plus complexe. Si tel fut globalement le cas jusqu'à l'indépendance du Ciskei, le gouvernement mis en place s'est arrogé le droit de propriété de l'ensemble des terres, seuls des droits d'usage collectifs (par l'intermédiaire des headmen ${ }^{12}$ ) ou individuels (baux emphytéotiques) étant accordés.

Les déplacements de population ont marqué toute l'histoire de la région : qu'il s'agisse de ceux, forcés, des black spots, villageois et agriculteurs privés noirs de l'ethnie Xhosa résidant dans les «zones blanches» du corridor ou d'autres régions du pays, ou des agriculteurs blancs expropriés du Ciskei, lors de son indépendance. La pression démographique qui en a résulté dans l'ancien Ciskei a renforcé l'attrait pour les revenus extra-agricoles via la migration, d'autant que l'agriculture noire n'était pas en mesure de concurrencer le secteur commercial blanc hautement subventionné. Cette pression et les dérégulations des mécanismes locaux de gestion des communs résultant des afflux de population seraient également à l'origine de phénomènes érosifs préoccupants et d'une déprise agricole généralisée. Cette dernière s'explique par l'attrait des activités extra-agricoles, mais aussi par l'absentéisme des producteurs auxquels l'État du Ciskei a attribué les terres des fermiers blancs expropriés. Par ailleurs, dans les quelques villages noirs qui subsistent dans le corridor, les conflits avec les propriétaires des fermes environnantes se sont développés depuis 1994.

12. Chefs traditionnels.
2. L'industrialisation, l'emploi urbain et les migrations.La localisation par rapport à la ville et aux sites industriels, les axes de communication et les systèmes de transport structurent fortement l'espace. En effet, la ville offre, entre autres, des sources d'emploi dans les secteurs secondaire et tertiaire et l'accès aux services administratifs, de santé et d'éducation. La distribution spatiale de la population est très inégale. Elle se concentre essentiellement dans le continuum territorial SE-NO de la zone urbaine et «rurbaine». Cette situation résulte des déplacements forcés de population et de la politique d'aide fiscale aux industries dans l'ancien Ciskei, comme à Dimbaza. La fin de l'apartheid s'est traduite par l'arrêt de ces aides et pourrait entraîner une baisse des activités.

La croissance urbaine et l'industrialisation ont modifié les phénomènes migratoires, qu'il semble possible de classer en trois catégories. La première correspond aux déplacements quotidiens depuis la zone «rurbaine» (dans une moindre mesure périurbaine) vers les zones urbaines et industrielles voisines pour aller travailler. La seconde est un processus de migrations successives par étapes : un jeune de la famille d'une zone communale reculée migre dans un premier temps vers la zone rurbaine, plus tard vers un township, se rapprochant ainsi progressivement du marché de l'emploi. Mais la multilocalisation de la famille reste de mise. Même inhabitée, la maison du village d'origine, en zone communale, reste occupée. La troisième correspond aux migrations des zones communales reculées vers les centres urbains et industriels situés à distance (Johannesburg, Le Cap). Les migrants ont pour habitude d'envoyer à leur famille une partie de leurs revenus. Cependant, le retour au village de mineurs licenciés au cours des dernières années est fréquent.

En fonction de la localisation par rapport à la ville ou au bourg rural et des conditions de transport, on peut donc distinguer plusieurs zones concentriques autour des bassins d'emploi et des axes de communication (fig. 3) : l'auréole rurbaine, en quelque sorte des dortoirs ruraux pour la main-d'œuvre urbaine; l'auréole périurbaine; les zones communales reculées. Dans chaque cas, les caractéristiques démographiques, les flux migratoires, la structure des revenus, les fonctions attribuées à la production agricole et la force de travail qu'elle emploie sont spécifiques et interdépendants. Dans les zones communales reculées, 
les ressources naturelles jouent un rôle d'autant plus sensible que, localement, l'agriculture représente souvent l'unique activité productive. Tel est le cas des vallées au nord au niveau de l'escarpement où les systèmes paysans sont restés très présents.

3. L'axe littoral.-En raison de son effet sur le régime pluviométrique, la proximité du littoral a marqué la distribution des espaces de l'apartheid et l'utilisation agricole des terres. Mais ce facteur joue également sur la localisation des centres et des emplois urbains, des axes routiers, sur les conditions de transport et sur le développement des sites et activités touristiques. Les situations rencontrées sur l'axe littoral sont tout à fait spécifiques : place de l'initiative privée, des emplois domestiques et dans le secteur touristique, axes de communication majeurs.

4. L'aménagement du territoire et l'investissement de l'ancien État du Ciskei dans le domaine agricole.L'aménagement de l'espace rural par l'ancien gouvernement du Ciskei s'est traduit par la production d'un espace «mité» où l'on peut différencier:

- des zones où les investissements ont été substantiels dans le domaine agricole (mise en place d'infrastructures d'irrigation ou de fermes d'État à forte utilisation de capital et de main-d'œuvre); partout, les services de l'État ont été omniprésents et leur disparition après 1994 pose de sérieux problèmes fonciers, de valorisation de l'investissement et de transfert à des opérateurs privés, sans évoquer le non-paiement des salaires des très nombreux fonctionnaires;

- des zones où l'expropriation des producteurs commerciaux s'est traduite par un abandon relatif de la production agricole; l'embroussaillement y est considérable, alors que, dans les villages communaux voisins, la pression sur les

ressources est très forte, l'érosion et la déprise agricole sont intenses ${ }^{13}$ et les conflits fonciers s'accentuent;

- le maintien de fermes commerciales à proximité du littoral;

- la création ou le maintien de forêts domaniales, principalement dans les régions accidentées du Nord de la zone d'étude, à des fins commerciales ou écologiques;

13. L'abandon du labour au tracteur, financé par les services publics, a été maintes fois signalé comme l'un des facteurs expliquant la déprise agricole. 


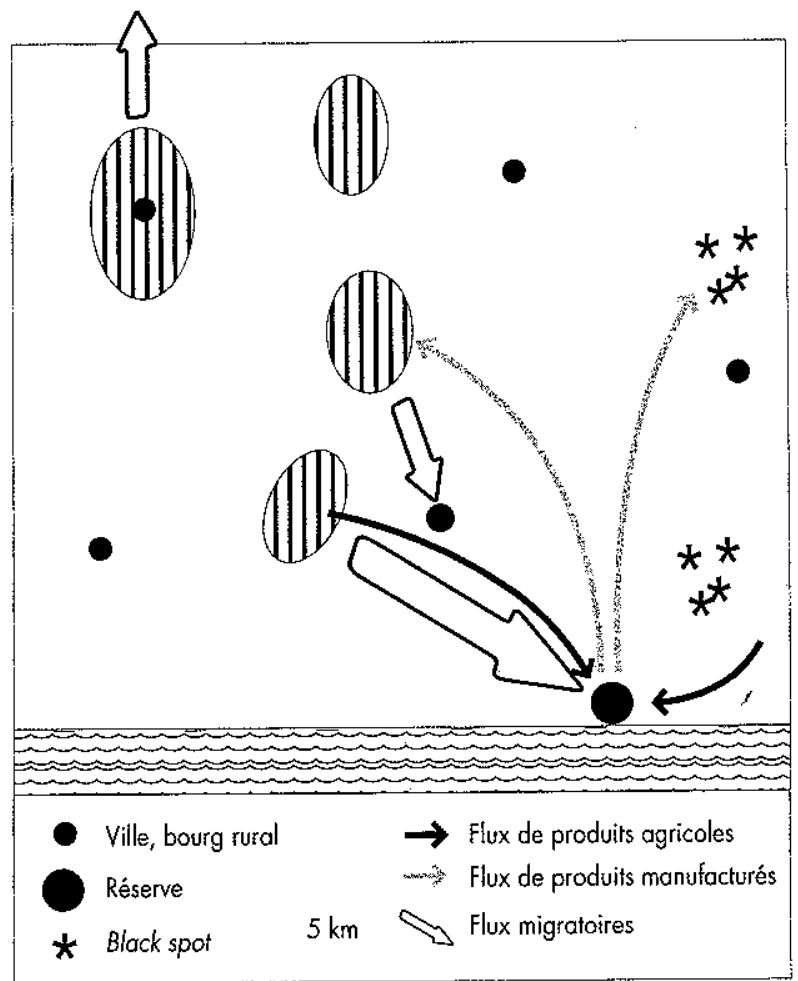

Fig. 5.-Organisation de l'espace rural dans la zone d'étude entre 1913 et 1950.

- l'investissement en zone urbaine et rurbaine (routes, électricité, adduction d'eau, subvention des industries, etc.), renforçant l'attrait de la ville et l'intensité des phénomènes migratoires.

Cette politique s'est ainsi traduite par la coexistence de poches d'intensification agricole où les problèmes se multiplient depuis 1994, et de zones marginales où la déprise est considérable. Après la disparition «brutale» de cet État, le bilan écologique, économique et social semble bien lourd.

\section{Reconstifution de l'histoire de la zone d'étude}

Les structures constitutives de l'espace se sont mises en place au cours de l'histoire mouvementée de la région. À partir des informations recueillies au cours des enquêtes, de la caractérisation de la diversité et d'une étude bibliographique, il est possible d'identifier les périodes clés qui ont façonné l'organisation spatiale actuelle.
1. 1913-1950: les bases d'une structuration ségrégée de l'espace (fig. 5).-- Le Natives' Land Act de 1913 représentait l'aboutissement d'un long processus historique ${ }^{14}$ entamé dès 1830 dans le Ciskei : la dépossession des terres africaines au profit des Blancs. Cette législation, clef de voûte des futures politiques d'apartheid, offrait aux Noirs un accès permanent au foncier dans les seules réserves mises en place à cet effet (environ $7 \%$ du territoire sudafricain à l'époque). La législation prévoyait aussi l'extension aussi large que possible du mode de tenure individuel dans les réserves. Elle tendait à limiter les formes de métayage d'exploitants noirs sur propriétés blanches au seul métayage en travail, abolissant le métayage sous forme monétaire ou en nature. L'objectif intrinsèque du Natives' Land Act était de créer des réservoirs de main-d'œuvre (les réserves) pour les mines, fermes, usines et foyers de l'Afrique du Sud blanche (Bundy, 1988).

Le Natives' Land Act a ainsi figé l'occupation de l'espace entre Blancs et Noirs. Le territoire de l'ancien Ciskei dépendait de la Province du Cap, où les Noirs ont conservé le droit d'acquérir de la terre jusqu'en 1936. Les exploitations noires en propriété privée représentaient à l'époque $12 \%$ de la surface occupée par la population noire. Elles étaient notamment concentrées dans le Magisterial District de Komga, dans le futur corridor blanc, où terres blanches et noires sont longtemps restées imbriquées. L'application du Natives' Land Act s'est contentée d'entériner une distribution spatiale existante, fruit des guerres de frontière. Cependant, les modifications des formes de métayage entre Noirs et Blancs firent refluer une population nombreuse vers les terres de la réserve. Dès 1916, l'État prit conscience qu'il fallait agrandir le territoire de la réserve et apposa ainsi une première empreinte ${ }^{15}$ sur l'espace.

L'application du Natives' Land Act entraîna l'organisation de migrations saisonnières de la population noire. Les jeunes affluaient vers le Gauteng (mines d'or), l'État libre (mines de

14. Le $\mathrm{XIX}^{\mathrm{e}}$ siècle a été marqué par neuf guerres, les Frontier Wars entre les colons britanniques et les chefferies xhosa pour le contrôle du territoire. La supériorité militaire des Britanniques a permis l'appropriation de grandes portions de territoire par les colons.

15. Ceci est la première empreinte spatiale «planifiée» des pouvoirs publics, même si les multiples guerres qui secouèrent la région au Xxx ${ }^{e}$ siècle avaient déjà considérablement marqué l'espace. 


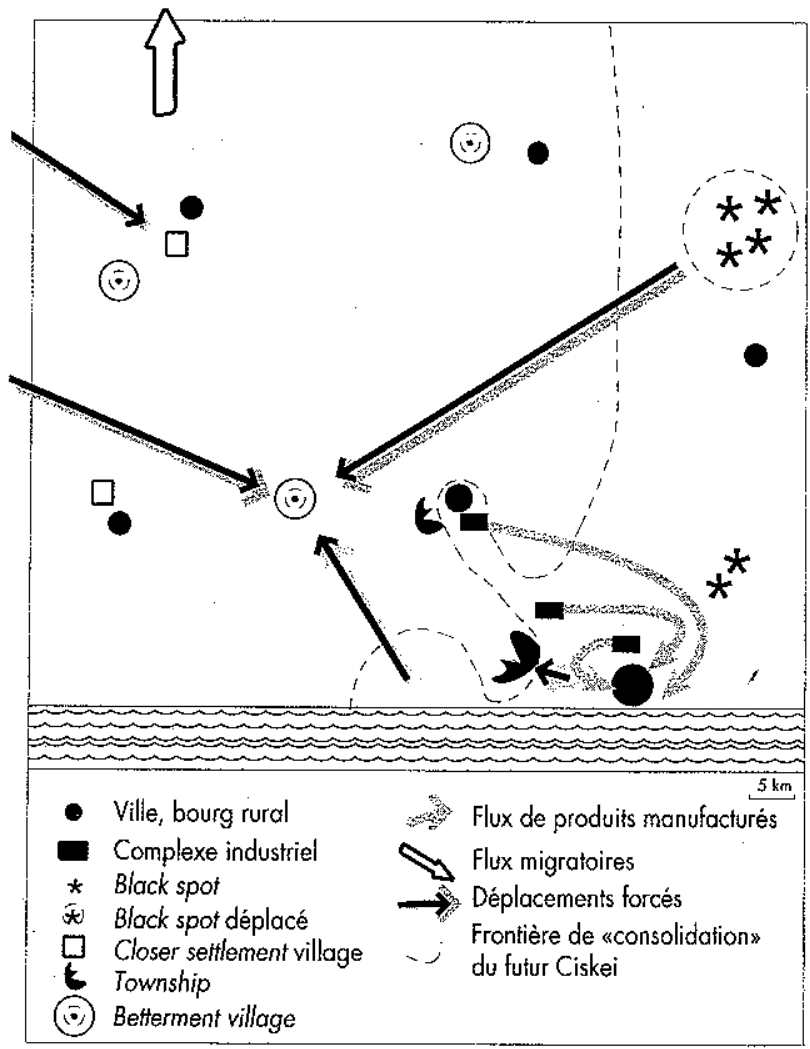

Fig. 6.--Organisation de l'espace rural dans la zone d'étude entre 1950 et 1980.

diamants) et les mines de charbon du Nord de la province du Cap de l'Est, les hommes d'âge mûr vers les activités non minières comme le travail dans les villes de la côte (East London, Port Elizabeth) et de l'intérieur (Stutterheim, etc.) et dans la construction des chemins de fer et des ports. Par ailleurs, de nombreuses familles noires travaillaient et demeuraient dans les exploitations blanches de la région. Les trading stores, magasins ruraux gérés par les Blancs, représentaient le pilier économique de la vie rurale. Véritables compradores et relais de la monétarisation des campagnes, ils assuraient aussi, moyennant de larges commissions, le recrutement de la main-d'œuvre pour le travail migrant.

East London s'était affirmé dès la fin du XIX ${ }^{\mathrm{e}}$ siècle comme le pôle économique de la région (Switzer, 1993). L'un des rares ports naturels de la côte, il fut établi en 1846 comme base arrière pour l'approvisionnement des garnisons britanniques pendant les guerres de Frontière. La ville s'est développée grâce aux capitaux du boom diamantifère. Jusqu'en 1950, East London vivait essentiellement de ses activités portuaires, s'affirmant vite comme l'un des grands centres d'exportation de produits agricoles (laine, peaux, maïs, etc.) et d'importation de produits manufacturés pour les populations noires. La ville reçut très tôt une forte immigration noire qui permit le développement de ses infrastructures.

2. 1950-1980, l'organisation d'un espace amené à être indépendant : le Ciskei (fig. 6).-- Les élections de 1948 virent l'arrivée au pouvoir du National Party. Sa politique agraire s'appuyait sur le renforcement de l'inégalité de la structure agraire établie par les Land Acts ${ }^{16}$, sur la poursuite de la colonisation agricole et sur le développement de l'agriculture blanche.

Créé à la fin des années 1940 à la périphérie de King William's Town pour abriter les populations sans-terre, le township de Zwelitsha («nouvelle ère» en xhosa) fut ainsi le premier bassin de main-d'œuvre industrielle mis en place dans une réserve sud-africaine. L'emploi était assuré par la construction d'une usine textile financée par des capitaux britanniques. Ce projet pilote a par la suite servi de modèle pour le développement économique et l'organisation spatiale des réserves, évitant ainsi un exode rural trop fort vers les villes, et plaçant la population noire à proximité des sources d'emploi.

Les années 1960 furent marquées par une très forte immigration vers les réserves, reposant sur quatre facteurs. La concentration foncière et la mécanisation croissante des fermes blanches entraînèrent des licenciements massifs d'ouvriers agricoles. La zone s'étendant du Cap à la Fish River fut déclarée zone d'emploi préférentiel métis. Les Noirs établis dans cette région étaient par conséquent systématiquement déplacés vers les réserves. Les populations noires vivant aux abords d'East London furent renvoyées vers le nouveau township de Mdantsane, à quelques kilomètres. Enfin, la majorité des propriétaires noirs, très

16. Le Native Trust and Land Act de 1936 était venu compléter le Natives' Land Act précédemment cité et permettait de libérer 6Mha supplémentaires pour les réserves qui représentaient alors $13,7 \%$ du territoire. 
minoritaires au cœur des zones blanches (black spots), furent déplacés de force vers les réserves.

Devant la dégradation de la situation dans les réserves, les recommandations de la commission Tomlisson (1955) ${ }^{17}$ furent mises en œuvre dans les années 1960-1970. Elles prirent la forme de programmes de villagisation ou Betterment Schemes dont l'objectif était de faire face à l'augmentation considérable de la densité de population, implicitement de veiller à la reproduction de la main-d'œuvre. Jusqu'alors, l'habitat des ruraux était dispersé sur les interfluves. Les agriculteurs cultivaient leurs champs dans les fonds des vallées, près des cours d'eau, et faisaient pâturer le bétail sur les versants et dans les forêts. Les programmes de Betterment ont regroupé l'habitat en créant de nouveaux villages et ont imposé une nouvelle localisation des parcelles cultivées sur les glacis. Ils s'accompagnaient de mesures rigides d'utilisation de la terre $^{18}$ et de réduction obligatoire de la taille des troupeaux. Même s'il fut diversement appliqué, le Betterment Planning a entrâné une recomposition considérable des espaces locaux et une dérégulation des mécanismes de gestion des communs (Lasbennes, 1998). Il s'est accompagné d'une séparation entre les populations agricoles et non agricoles. Seuls les ménages possédant des ressources suffisantes en bétail et terres pouvaient bénéficier du Betterment, les autres étaient déplacés vers les Closer Settlement Villages, véritables townships rurbains, réserves de main-d'œuvre situées le plus souvent le long des voies de communication (De Wet, 1995).

La politique de développement industriel s'affirma dans les années 1970. De nouvelles industries, attirées par les privilèges fiscaux accordés par le gouvernement sud-africain pour promouvoir la création d'emplois, s'implantèrent autour d'East London et de King William's Town. Les matières premières étaient généralement importées via East London et les produits manufacturés réexportés vers les

17. La Commission Tomlisson, mise en place par le gouvernement, s'est penchée pendant 5 ans sur la situation dans les réserves. Elle a formulé des recommandations en termes de politique économique.

18. Une délimitation stricte entre terres cultivables, terres de parcours et zones résidentielles a été mise en place. Le pâturage rotationnel était imposé et le passage du bétail d'un camp à l'autre était contrôlé par un agent de l'administration. grands centres urbains du pays. Avec la perspective d'une réserve «indépendante», le gouvernement sud-africain procéda dès 1975 à son «remembrement». Un territoire d'un seul tenant fut constitué. Le futur Ciskei se vit ainsi attribuer des terres agricoles dans les districts de Peddie, King William's Town, Keiskammahoek et East London.

\section{1980-1994 : l'État indépendant du Ciskei et son héritage} (fig. 7). - Lors d'un référendum tenu le 4 décembre 1980 le Ciskei opta pour l'indépendance. Dès l'origine considéré comme une entité non viable ${ }^{19}$, le Ciskei s'embarqua toutefois dans un vaste programme de développement économique ${ }^{20}$ reposant sur deux axes : l'installation d'industries et le développement agricole sur la base de grands projets à forte utilisation et concentration de capitaux.

Le développement de la zone industrielle (métallurgie, bois et textile) de Dimbaza créa un nouveau bassin d'emploi au coeur du bantoustan. Il s'accompagna de l'apparition d'un nouveau township dans son voisinage. Les restrictions de circulation et de migration entre le Ciskei et l'Afrique du Sud se traduisirent par des mouvements de main-d'œuvre pendulaires journaliers ou hebdomadaires. En raison du déclin prononcé des activités agricoles, se développèrent des stratégies familiales de multilocalisation et les townships de Zwelitsha et Mdantsane explosèrent pour former une immense cité dortoir entre King William's Town et East London.

D'ambitieux et coûteux projets de développement agricole furent mis en place pour tenter d'enrayer la déprise agricole. Des périmètres irrigués furent dessinés le long des principaux cours d'eau et Ulimocor, entreprise agricole étatique, assurait la gestion des fermes blanches expropriées les plus productives (plantations d'ananas, exploitations laitières). D'autres exploitations acquises lors du «remembrement» étaient attribuées à des dignitaires du régime, qui

19. La Quail Commission commanditée par le gouvernement sud-africain en 1980 se pencha sur les possibilités économiques d'un Ciskei indépendant.

20. On peut signaler aussi la création ex nihilo, à quelques kilomètres de King William's Town, de Bisho, la capitale de prestige du nouvel État. Bisho est désormais la capitale de la province du Cap de l'Est. 
les laissèrent souvent à l'abandon. Cette politique interventionniste aboutit à l'espace rural mité que l'on rencontre aujourd'hui.

\section{Conclusion}

La méthode de zonage expérimentée s'appuie sur la description de la diversité et la recherche des ordres sousjacents. C'est à partir de la diversité que sont mises en évidence les dynamiques des espaces ruraux. La recherche confirme l'intérêt de recourir aux dires d'acteurs, dont la capacité d'expertise se révèle extrêmement féconde. Cette richesse contraste avec le peu d'informations secondaires disponibles. L'intégration par ces témoins des dimensions spatiales, techniques, économiques et sociales des processus de production et de consommation fournit un support structurant pour l'analyse de la dynamique des espaces ruraux. La diversité spatiale est extrêmement marquée dans la zone d'étude. Elle s'explique en partie par l'histoire de la région dont l'empreinte spatiale est considérable. Le zonage a permis d'en identifier les grands traits.

La politique d'apartheid a différencié et structuré les espaces des différents groupes ethniques. Des espaces bien délimités et dissymétriques en sont issus. Néanmoins, l'organisation de chacun dépend étroitement de son intégration à l'autre. La distribution spatiale de la population et des centres urbains et industriels et les mouvements migratoires qui structurent l'espace rural de l'ancien Ciskei répondent à un phénomène de gravité dont le centre correspond à la ville d'East London. Il existe une hiérarchie de centres secondaires (villes) et tertiaires (bourgs ruraux, comptoirs, centres administratifs), gravitant autour d'East London et reliés entre eux par un quadrillage constitué des principaux axes de communication. Cette organisation se traduit par un maillage de l'espace où chaque maille (USH) possède des caractéristiques spécifiques et des limites qui correspondent rarement à celles des entités administratives. Les flux d'hommes et de femmes, de marchandises, de capitaux et d'informations révèlent le fonctionnement d'interdépendances entre ces mailles et mettent en évidence, à l'image des processus migratoires, l'existence de réseaux complexes et enchevêtrés. La reconnaissance de ces réseaux permet de raisonner le développement de la région en dehors des limites historiques de l'apartheid.

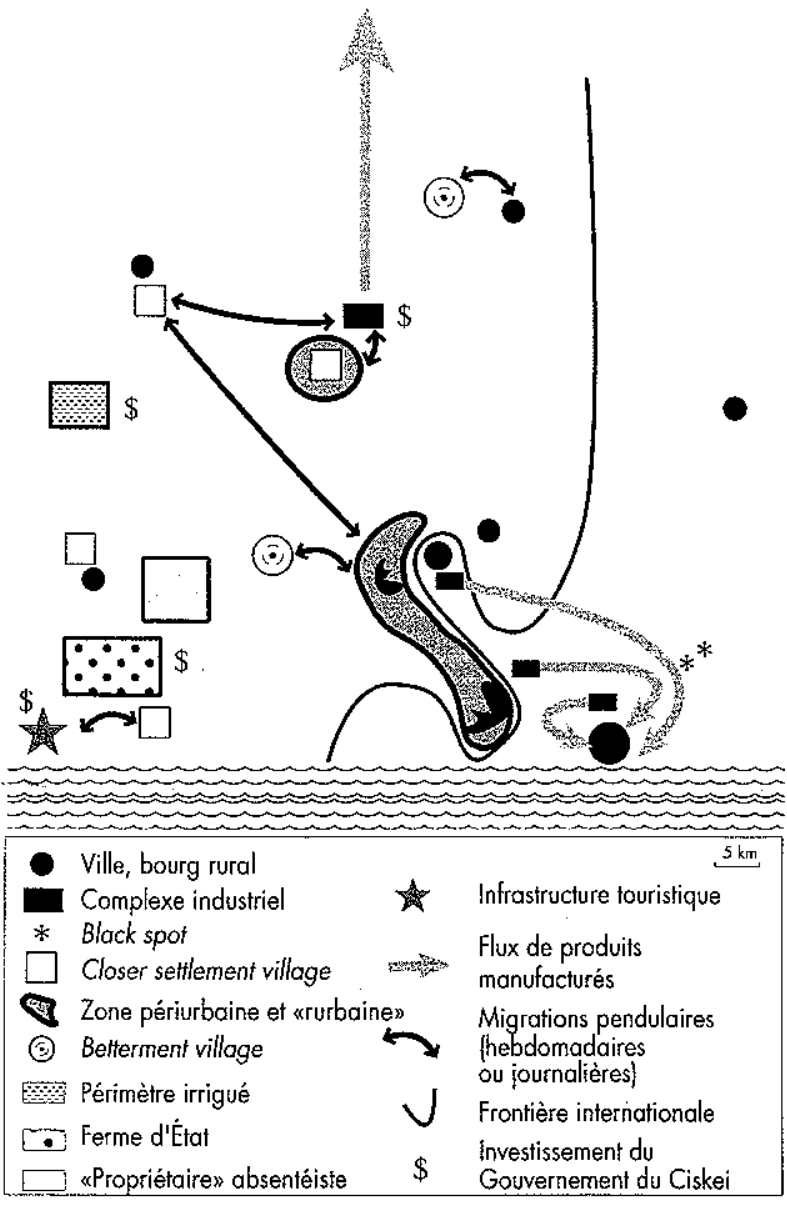

Fig. 7.-Organisation de l'espace rural dans la zone d'étude entre 1980 et 1994.

Les changements politiques intervenus depuis 1994 ont peu modifié cette organisation. Ils sont récents. Le poids de l'histoire est considérable et les réformes sont difficiles à mettre en œuvre. Par contre, dans la plupart des mailles, les situations ont considérablement évolué : déprise agricole ici, retrait de l'État de l'ensemble du processus de production et transfert aux opérateurs privés là, conflits fonciers et intensification des transactions ailleurs, réforme agraire «informelle», etc.

Dans un contexte marqué par une telle différenciation, le nouveau découpage politique et administratif cherche à transcender, à bousculer volontairement cette marque de l'histoire. Le district d'Amatola est constitué d'entités des 
anciens bantoustans et du corridor. La politique d'aménagement du territoire et d'appui au développement rural que souhaite mettre en place cette collectivité territoriale est tout à la fois une tâche difficile et un enjeu majeur. Elle souligne la volonté de ne pas raisonner le développement agricole et rural dans les structures et les limites spatiales issues de l'apartheid.

Par la construction de nouvelles représentations et de nouveaux modèles des réalités, par la production d'informations opérationnelles pour susciter la réflexion et le débat, la recherche peut jouer un rôle important (Caron et al., 1997). Elle peut contribuer à la formalisation de projets individuels et collectifs, en participant à l'élaboration d'un système d'aide à la décision. Outre la production et la diffusion d'informations, l'engagement de la recherche doit s'accompagner de l'expérimentation de mécanismes spécifiques de concertation, d'animation et de médiation, afin que la carte ne devienne pas le support de pratiques technocratiques de planification. Un engagement dans la durée est nécessaire. En effet, les limites tracées sur une carte figent les représentations alors qu'elles évoluent et se transforment. De nouvelles USH peuvent rapidement apparaître, disparaître, s'étendre ou se réduire. Leurs caractéristiques changent. Le zonage ne représente qu'une photographie à un instant donné mais cette image marque les esprits. Comme l'affirme Roger Brunet (1990), « une fois produites, les images durent bien plus longtemps que les réalités auxquelles elles se sont substituées ». L'enjeu est désormais de mettre en place un système de suivi des situations et d'actualisation des informations, sur la base d'indicateurs de changement mesurables et identifiés grâce à l'analyse présentée ici. La mise en place d'un tel suivi soulève de nouvelles questions méthodologiques, dans la mesure où les USH ne correspondent pas aux unités habituelles de recensement.

\section{Bibliographie}

Bonnal P., CARon P., LaURENT C. (1998). Agriculture familiale dans l'ex-Ciskei. Discussion d'une démarche d'analyse et d'intervention. Rapport de mission en Afrique du Sud du 10 au 24-01-98. Montpellier: CIRAD-TERA, $21 \mathrm{p}$.
BRUNET R. (1987). La Carte, mode d'emploi. Paris/Montpellier: Fayard/Reclus, $270 \mathrm{p}$.

Brunet R., Dollfus O. (1990). Mondes nouveaux. Vol. 1 de la Géographie Universelle. Paris: Belin/Reclus, $551 \mathrm{p}$.

BUNDy C (1988). The Rise and Fall of the South-African Peasantry. Londres: David Philip, $276 \mathrm{p}$.

CARON P. (1997), «Le zonage régional à dires d'acteurs. Connaître, représenter, planifier et agir, une méthodologie expérimentée dans le Nordeste du Brésil ». In Clouer Y., TONNEAU J.-P., dir. Quelle géographie au CIRAD? Document de travail du CIRAD-SAR, n 10 , p. 145-156.

CARON P. (1998). Zonage à dires d'acteurs dans le District d'Amatola en Afrique du Sud. Rapport de mission du 13 au 30 avril 1998 dans la Province de l'Eastern Cape. Montpellier: CIRAD-TERA, $53 \mathrm{p}$.

Caron P., Sabourin E., Sautier D., Silva P.C.G. da, Tonneau J.P. (1997). «À la recherche de l'opérationnalité : le cas de

.. l'agriculture familiale dans le Nordeste du Brésil». In GASTELlu G., MARChal J.-Y., dir. La Ruralité dans les pays du Sud à la fin $d u X X^{e}$ siècle. Paris: ORSTOM Éditions, coll. «Colloques et Séminaires», p. 639-662.

DBSA (1991). Eastern Cape : Statistical Macroeconomic Review. Halfway House: DBSA, 183 p.

DE WET C. (1995). Moving Together Drifting Apart. Johannesburg: Witwatersrand University Press, $253 \mathrm{p}$.

LE MoIGNe L. (1990). La Modélisation des systèmes complexes. Paris: Dunod, $178 \mathrm{p}$.

LASBENNES F. (1998). Modes de gestion des ressources renouvelables collectives à l'échelle du village dans l'ancien Ciskei, Afrique du Sud. Dijon: ENESAD, Projet d'ingénieur non publié, $112 \mathrm{p}$.

LHOPITALLIER L. (1998). «Les mosaïques culturelle et foncière de l'ancien bantoustan du Ciskei (ou les problèmes d'une « campagne urbaine »)». Géographie et cultures, n²8, p. 31-46.

Lhopttallier L., Peuch-Lestrade E. (1997). «Le désert agricole de l'ancien Ciskei : le poids de l'histoire». Johannesburg: Institut Français d'Afrique du Sud, Newtown Zebra, n 7, p. 2-7.

Ministry of Provincial AfFairs and Constitutionnal GoverNMENT (1998). White paper on local government. Pretoria: Government Printer, $150 \mathrm{p}$.

Perrot C., LandaIS E. (1993). «Comment modéliser la diversité des exploitations agricoles». Montpellier: CIRAD-SAR, Cahiers de la Recherche-Développement, $\mathrm{n}^{\circ} 33$, p. 24-40.

Peuch-Lestrade E. (1997). Étude des systèmes agraires $d u$ district de Keiskammahoek. Paris: INA-PG, Rapport de fin d'études non publié, $42 \mathrm{p}$.

SwITZER L. (1993). Power and resistance in an African Society: the Ciskei Xhosa and the making of South Africa. Pietermaritzburg: University of Natal Press, $452 \mathrm{p}$. 